\title{
The Effect of an Asymmetric Energy Window on Bone Scintigraphy Image Quality
}

\author{
Joana do Mar F. Machado ${ }^{1}$, Susan Doshi ${ }^{1}$, Ruth Smith ${ }^{2}$, Martyn Evans ${ }^{1}$, Richard N.J. Graham ${ }^{1}$, Stewart Redman ${ }^{1}$, \\ and David Little ${ }^{1}$ \\ ${ }^{I}$ Nuclear Medicine Department, Royal United Hospitals Bath, Combe Park, Avon, United Kingdom; and ${ }^{2}$ Auckland District Health \\ Board, Auckland City Hospital, Grafton, Auckland, New Zealand
}

\begin{abstract}
Bone scintigraphy is one of the most common nuclear medicine tests. Previous work investigated the effectiveness of an asymmetric window (ASW) for planar bone scintigraphy using simulation and phantom data. Phantom studies concluded that the ASW improved both the resolution and the contrast-tonoise ratio when imaging objects with high scatter. The aim of this study was to confirm this improvement increased image quality in patients. This study also investigated whether the differences between a symmetric window (SW) and an ASW depended on body mass index. Methods: Fifty-eight patients had 2 scans: a standard scan using an SW of $140 \mathrm{keV} \pm 10 \%$ and a scan using an ASW of $140 \mathrm{keV}+10 \%$ and $-7.5 \%$. Three readers independently compared the 2 image sets and scored them using a 5-score scale (ranging from 1 = ASW better [clinically important] to $5=\mathrm{SW}$ better [clinically important]). Scores from all radiologists were pooled and analyzed statistically. A $P$ value of less than 0.05 was considered statistically significant. Results: In 93 cases (53\%), the readers scored the ASW images better than the SW images. In 5 cases (3\%), the ASW images were preferred, with the difference considered clinically important; there were no cases in which the SW was similarly preferred. For the sign test, we determined whether the total of 93 scores of 1 or 2 (ASW preferred) was significantly different from the 15 scores of 4 or 5 (SW preferred). The $P$ value was less than 0.00001 , demonstrating that the difference was significant. Conclusion: In patients undergoing bone scintigraphy, ASW provided an improvement in image quality that in some cases was judged clinically important.
\end{abstract}

Key Words: bone scintigraphy; asymmetric energy window

J Nucl Med Technol 2020; 48:46-50

DOI: 10.2967/jnmt.119.233577

B one scintigraphy is one of the most common nuclear medicine procedures performed in the United Kingdom and in the United States, representing $29.6 \%$ of nuclear medicine procedures in 2003/2004 (the most recently available national

Received Jul. 8, 2019; revision accepted Aug. 30, 2019.

For correspondence or reprints contact: David Little, Royal United Hospitals Bath, Combe Park, Avon BA1 3NG, U.K.

E-mail: davidlittle@nhs.net

Published online Oct. 11, 2019.

COPYRIGHT (C) 2020 by the Society of Nuclear Medicine and Molecular Imaging. survey) and 17\% in $2011(1,2)$. Although bone scintigraphy is considered to be one of the most reliable nuclear medicine examinations (3), the detection of lesions is affected by image quality, which depends on several different variables, such as age $(4,5)$, time between dose and scan $(6,7)$, radiopharmaceutical (8), distance between the patient and the collimator (3), and body mass (9).

Scatter, an important process in nuclear medicine imaging, usually refers to Compton scattering. A $\gamma$-ray interacts with matter (e.g., the patient), decreasing the energy of the $\gamma$-ray and deflecting it from its original path (10). Scattered photons are one of the main defects that degrade the quality of nuclear medicine images and sometimes represent more than half the total counts in clinical imaging $(11,12)$. Scatter deflects photons, with a concomitant loss of energy leading to blurring, loss of image contrast, and, consequently, poor image quality $(13,14)$.

Detector technology has advanced rapidly, but there are still profound challenges in diagnostic imaging with regard to scatter radiation, particularly in obese patients. An increased body mass attenuates photons by absorbing and scattering them within the soft tissue, leading to a reduced signal-tonoise ratio, increased scatter, and nondiagnostic results $(9,15)$.

Most nuclear medicine departments apply predefined protocols designed to exclude scattered photons through use of a suitable energy window, which is an accepted trade-off between minimizing the acceptance of scattered photons and maximizing the acceptance of unscattered radiation (16).

The photopeak is the peak formed when an incident $\gamma$-ray is completely absorbed in the crystal because of the photoelectric effect (17). The event detection necessary to produce an image occurs in an energy window around the photopeak energy, which is typically a $15 \%$ or $20 \%$ symmetric window (SW) centered over the $140-\mathrm{keV}$ photopeak of ${ }^{99 \mathrm{~m}} \mathrm{Tc}$. This $\mathrm{SW}$ is equivalent to a window spanning 130 $151 \mathrm{keV}$ and $126-154 \mathrm{keV}$, respectively $(18,19)$. An asymmetric window (ASW) is produced when the photopeak energy is not at the center of the window because the energy window has been shifted to the right side on the spectrum (20). An ASW can be used in practice to avoid lower-energy photons and to minimize the amount of scatter that is recorded (21). The number of counts collected is reduced, 


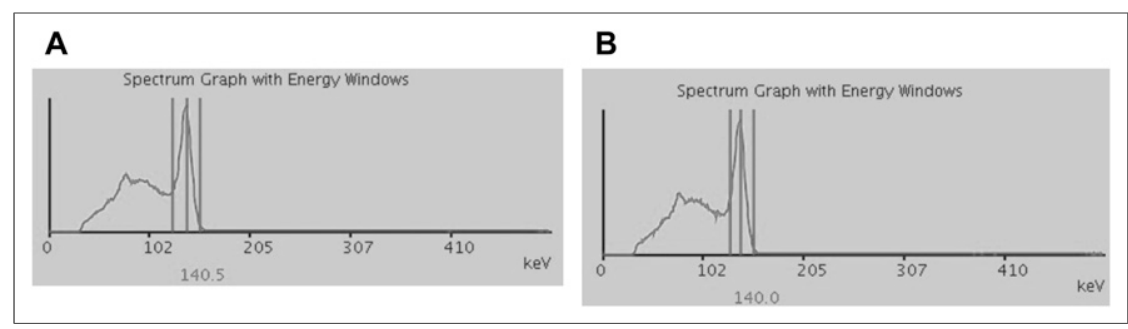

FIGURE 1. $y$-spectrum and photopeak parameters of ${ }^{99 m} \mathrm{Tc}$ isotope using SW (A) and ASW $(B)$ recorded during whole-body bone scintigraphy. Screenshots are from GE Healthcare Discovery NM/CT 670 workstation.

thus elevating noise, and scatter is still present but at a reduced level (22). Use of an ASW must be supported by a physicist who can help in determining the limits of asymmetry $(18,19)$. In this study, an ASW of $-7.5 \%$ and $+10 \%$ over the $140-\mathrm{keV}$ photopeak of ${ }^{99 \mathrm{~m}} \mathrm{Tc}$ was investigated, which is equivalent to a window spanning $129.5-154 \mathrm{keV}$.

Although attempts have been made to evaluate the effect of an ASW on contrast resolution (23), scatter and attenuation correction (24), and flood-field uniformity (25), there are no data about the use of an ASW to improve image quality in whole-body bone scans obtained on contemporary equipment. This study aimed to transfer the results of our phantom work to patients to confirm that image quality in whole-body bone scans is improved by using an ASW. Associations were examined between image quality scoring and body mass index (BMI).

\section{MATERIALS AND METHODS}

\section{Patient Population}

The study included 36 male and 22 female patients with an age range of 31-87 y who were seen from January 2014 to February 2016. Among the 58 patients, most presented with prostate cancer $(n=32)$ or breast cancer $(n=12)$. Patient weight averaged $79.8 \mathrm{~kg}$.

All patients underwent SW whole-body imaging first, followed by an ASW whole-body scan.

The patients were categorized into 4 groups according to BMI as defined by the National Health System: underweight (BMI < $\left.18.5 \mathrm{~kg} / \mathrm{m}^{2}\right)$, healthy weight $\left(\mathrm{BMI}=18.5-24.9 \mathrm{~kg} / \mathrm{m}^{2}\right)$, overweight $\left(B M I=25-29.9 \mathrm{~kg} / \mathrm{m}^{2}\right)$, and obese $\left(B M I=30-39.9 \mathrm{~kg} / \mathrm{m}^{2}\right)$.

Approval for the study was obtained from the Bristol Research Ethics Centre (institutional review board equivalent), and all subjects gave written informed consent. All procedures were in accordance with the 1964 Helsinki declaration.

TABLE 1

Five-Point Scoring System

\begin{tabular}{lc}
\hline \multicolumn{1}{c}{ Result of comparison } & Score \\
\hline ASW better (clinically important) & 1 \\
ASW better (not clinically important) & 2 \\
Neither preferred & 3 \\
SW better (not clinically important) & 4 \\
SW better (clinically important) & 5 \\
\hline
\end{tabular}

The datasets generated during or analyzed during the study are available from the corresponding author on reasonable request.

\section{Imaging Technique}

The study was done using a dual-head Infinia Hawkeye $\gamma$-camera (GE Healthcare), equipped with low-energy, high-resolution collimators and capable of simultaneous anterior and posterior acquisition. Quality assurance testing was conducted daily before scanning. A new uniformity map was acquired for the ASW acquisition.

A SW whole-body scan was obtained 2$3 \mathrm{~h}$ after injection of 546-640 MBq of ${ }^{99 \mathrm{~m} T c-h y d r o x y d i p h o s p h o-}$ nate as per the British Nuclear Medicine Society guidelines for bone scintigraphy (26). Patients were invited to empty the bladder before the first part of the acquisition. Directly after the SW whole-body scan, an ASW whole-body scan was performed.

The SW acquisition ranged from the top of the head to the bottom of the feet, and the ASW acquisition ranged from the top of the head to the knees. The field of view was shorter for the ASW than for the SW to reduce the acquisition time, as well as to avoid patient movement and, consequently, degradation of image quality.

The 2 sets of images were obtained in both anterior and posterior projections, with the patients supine and a hook-and-loop strap used to help them keep their arms by their sides. A pillow to

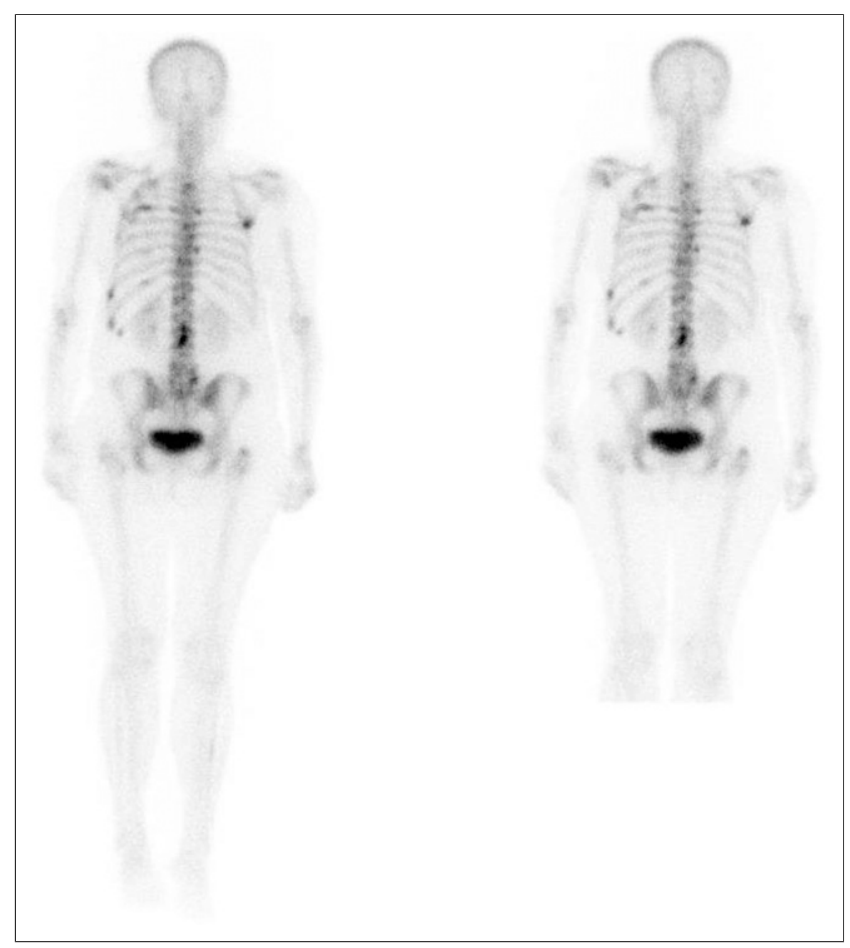

FIGURE 2. SW (left) and ASW (right) whole-body bone scintigraphy in breast cancer patient with coexistence of bone metastases. Widespread foci of abnormal tracer activity are seen within skull, thoracic spine, ribs, shoulders, lumbar spine, and proximal femora in keeping with widespread bony metastatic disease. All readers rated ASW as preferred but not clinically important. 


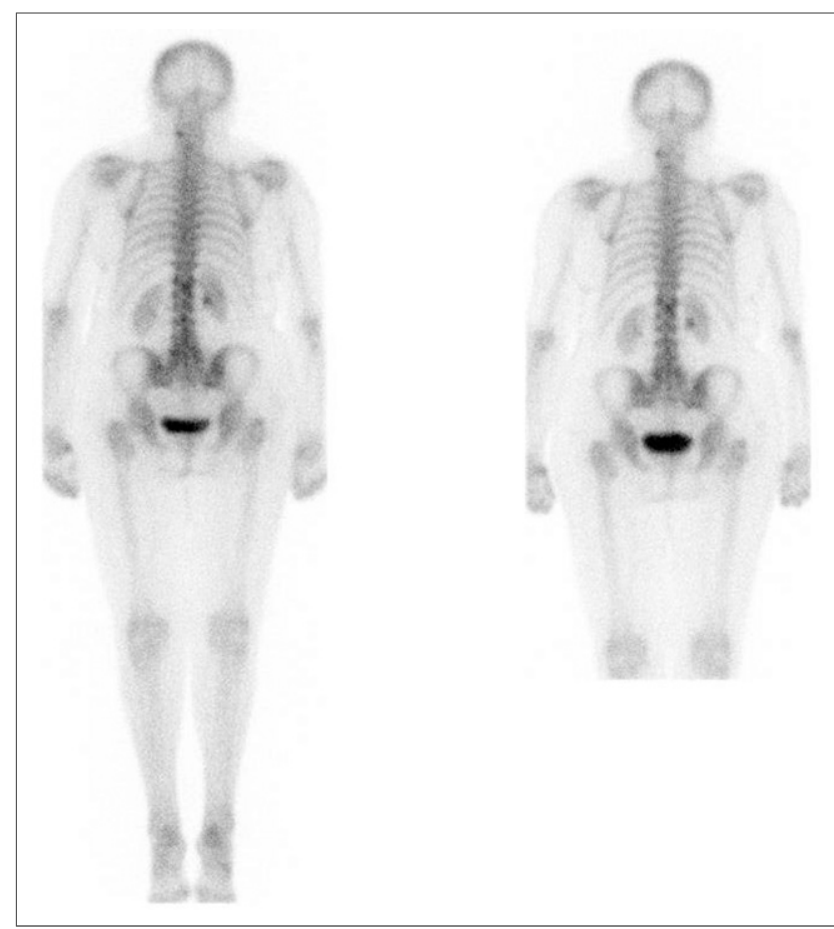

FIGURE 3. SW (left) and ASW (right) whole-body bone scintigraphy in breast cancer patient. Thoracolumbar junction region shows minor increased uptake that appears to correlate with degenerative change seen on radiography at T12-L1. One reader rated ASW as preferred but not clinically important. Another reader rated SW as preferred and clinically important because it potentially improves assessment of lumbar spine. Third reader rated neither as preferred.

support the head was used for the duration of the examination. Scan speed was $10 \mathrm{~cm} / \mathrm{min}$, exposure time was $240 \mathrm{~s}$, matrix size was $256 \times 1,024$, and zoom was 1.0. The energy window was defined relative to the photon energy peak of ${ }^{99 \mathrm{~m}} \mathrm{Tc}(140 \mathrm{keV})$ for both scans. The window width was set at $-10 \%$ and $+10 \%(126-154 \mathrm{keV})$ for the SW scan and $-7.5 \%$ and $+10 \%$ (129.5-154 keV) for the ASW scan, as illustrated in Figure 1.

After both scans were performed, the patients' weight and height were measured.

\section{Image Assessment}

To assess the impact of an ASW on clinical images, 3 experienced radiologists with a specialist interest in nuclear medicine who were unaware of the clinical information examined the scans using a dedicated image analysis workstation (Xeleris; GE Healthcare). A coding system was used to anonymize the patient data before image analysis.
The 2 whole-body images were displayed on the same screen and using the same color scale. Briefly, the visual evaluation consisted of comparing the intensity of abnormal uptake in the SW image with that in the ASW image and scoring the differences as in Table 1. BMI was not compared with image outcome

\section{Statistics Assessment}

We tested 2 hypotheses: that a preference for SW or ASW is equally likely (the null hypothesis) and that a preference for either SW or ASW is more likely.

The sign test was used for statistical analyses. In this test, a score of 1 or 2 indicated that ASW was preferred, and these 2 scores were added together to determine the total number for which ASW was preferred (N_A). A score of 4 or 5 indicated that SW was preferred; likewise, these 2 scores were added together to determine the total number for which SW was preferred (N_S). The sign test is a test of whether N_A is drawn from a binomial distribution $\left(\left[\mathrm{N} \_\mathrm{A} \sim \operatorname{bin}\left(\mathrm{N} \_t\right.\right.\right.$ tot, $\left.\left.q\right)\right]$, with N_tot $=$ N_A + N_S and $\mathrm{q}=0.5)$. An $\alpha$-value of 0.05 was used to determine whether a $P$ value indicated a significant result. The calculations were performed using a freely available online binomial calculator (http:// stattrek.com).

\section{RESULTS}

In total, 58 patients were enrolled and 116 images analyzed: $58 \mathrm{SW}$ and $58 \mathrm{ASW}$. The 3 independent radiologists scored the images according to the 5-point scoring system, resulting in a total of 174 scores.

Example images from the SW and ASW acquisitions are shown in Figures 2 and 3.

Table 2 and Figure 4 show the results of the image comparison. The data are ordinal, allowing calculation of median. There were 5 possible scores, with the middle score (3) representing no preference for either SW or ASW; most sets of images were assigned this score. Scores of 1 and 2 represented a preference for ASW, and scores of 4 and 5 represented a preference for SW; it is these scores that we analyzed using the sign test. In total, 93 scores were less than 3 , and 15 scores $(8.6 \%)$ were more than 3 . Use of the sign test to compare an N_A of 93 with an N_S of 15 gave a $P$ value of less than 0.000002 ; we therefore rejected the null hypothesis that ASW and SW are equally likely to be preferred.

Mean BMI was $27.7 \mathrm{~kg} / \mathrm{m}^{2}$. Figure 5 presents the imaging scores analyzed by patient BMI. Within the group that had scores of less than 3, the proportion of obese patients was similar to that of nonobese patients. A score of less than 3 was reported for $70 \%$ of healthy-weight patients and $92 \%$ of overweight patients, versus $85 \%$ of obese patients.

TABLE 2

Percentages of Comparison Scores for 3 Independent Readers

\begin{tabular}{|c|c|c|c|c|c|}
\hline \multirow[b]{2}{*}{ Radiologist } & \multicolumn{2}{|c|}{ ASW better } & \multirow[b]{2}{*}{ Neither preferred } & \multicolumn{2}{|c|}{ SW better } \\
\hline & Clinically important & Not clinically important & & Not clinically important & Clinically important \\
\hline 1 & 7 & 22 & 66 & 10 & 0 \\
\hline 2 & 7 & 57 & 24.1 & 12 & 0 \\
\hline 3 & 0 & 72 & 24 & 3 & 0 \\
\hline Overall & 2.9 & 50.6 & 37.9 & 8.6 & 0 \\
\hline
\end{tabular}




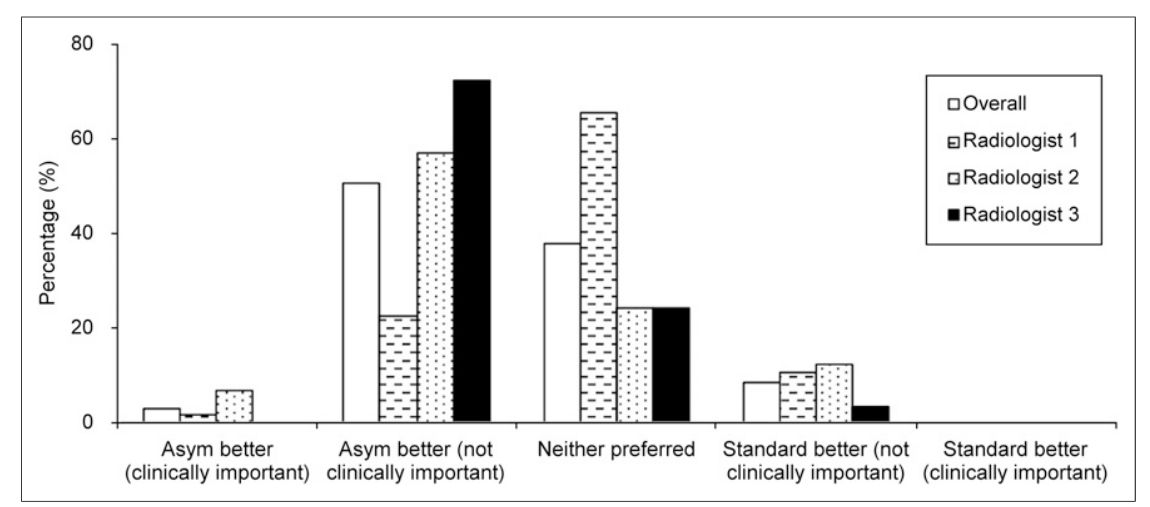

FIGURE 4. Bar graph comparing the pairs of images for all 58 patients. Results are expressed as radiologists 1,2 , and 3 against different imaging scores.

scans. The ASW images are still preferred despite the reduced overall counts. Increasing the acquisition time may increase the quality of images still further.

Shifting the ${ }^{99 \mathrm{~m}} \mathrm{Tc}$ window to the right has been shown to be effective in reducing scatter. In most cases in which a preference was expressed, an ASW was preferred. We did not randomize the order of the scans; we performed SW imaging first because that is the standard care and patients should be able to decline the second scan at any time. It is therefore possible that the ASW was preferred not

No significant differences across BMI categories were observed for the different imaging scores.

\section{DISCUSSION}

In this study, we evaluated the effectiveness of using an ASW in planar bone scans. To our knowledge, this study was the first to explore the impact of an ASW on image quality in planar bone scans obtained on contemporary equipment.

The imaging parameters may be further adapted to the clinical indication by decreasing the time between injection and imaging, reducing the acquisition time, or increasing the administered activity (14). The work presented here did not consider these factors.

As Asgari et al. (18) indicated, scatter fraction seemed to be lower with an ASW than with an SW in ${ }^{99 \mathrm{~m}} \mathrm{Tc}$ and ${ }^{153} \mathrm{Sm}$ imaging of a Solid Water (Sun Nuclear Corp.) slab phantom and Teflon (DuPont) bone phantoms. In an earlier study (23), Collier et al. found that contrast resolution was significantly improved by switching to an ASW in anterior views of the abdomen and lumbar spine $3 \mathrm{~h}$ after the injection of $740 \mathrm{MBq}$ of ${ }^{99 \mathrm{~m}} \mathrm{Tc}-$ methylene diphosphonate.

With ASW, the energy window is smaller and more counts will be rejected. To maintain the number of counts in the final image, one would have to increase the injected activity or the scanning time. However, in this case we used a fixed standard couch-speed for both the ASW and the SW

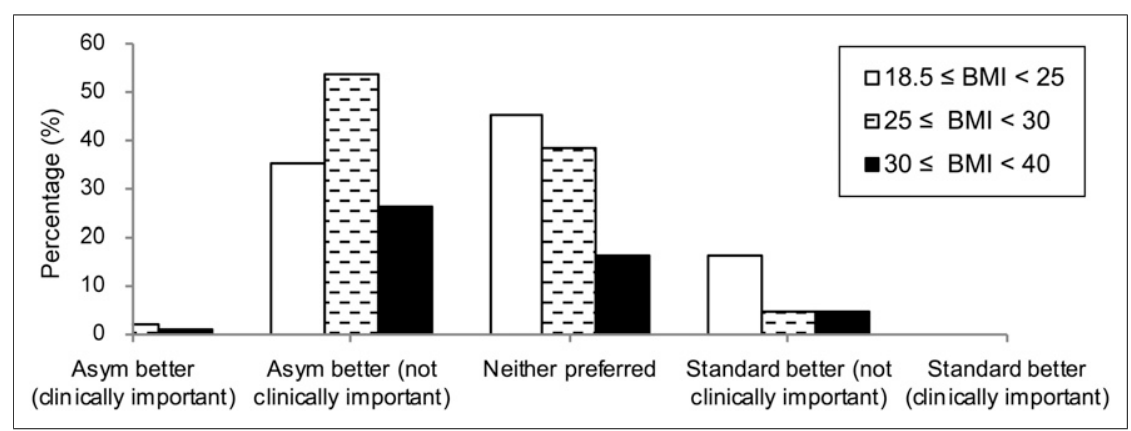

FIGURE 5. Bar graph comparing the pairs of images according to patient BMI. Results are expressed as BMI intervals against different imaging scores. because of its asymmetry but because of the systematic timing difference, leading to differences in, for example, uptake and washout. However, it is well established that whole-body images are usually acquired $2-5 \mathrm{~h}$ after injection. We therefore presume that the asymmetry is more likely to be the reason for the expressed preference of the ASW.

Obesity is well known to present challenges in various imaging modalities. Problems associated with dose to be administered, image acquisition time, and image noise are still being discussed in the literature. There are no clear conclusions about how we can overcome these challenges, but efforts have been made to investigate weight-based dosing and imaging time $(9,15)$. Our study found similar results in the different BMI groups, possibly because of the limited number of patients included. No comparisons were made between men and women or between different clinical indications. Moreover, the effects of obesity on image quality were not directly analyzed. An alternative approach would be to monitor differences in injected activity between the normal, overweight, and obese groups.

Our study had some limitations. Although we found a highly significant overall result, we did not find any statistically significant effect related to BMI. We suspect that a larger number of patients would be needed to demonstrate such an effect.

We did not undertake quantitative measurements of signal-to-noise ratio; such measurements might have disentangled the effect of uptake and washout.

We relied on qualitative comparison scores, not quantification of contrast as has been done in phantom work. This choice was because we are interested in clinical practice and the utility of the images to radiologists.

Finally, we decided on a shorter field of view for ASW to avoid motion. However, this shorter field of view should not be excluded as a possible confounder, 
because it prevented the scorers from identifying lesions in lower limbs and also made blinding the scorers impossible.

Nonetheless, after many years of clinical evaluation, bone scintigraphy continues to have a significant impact on patient management, mainly in the evaluation of metastatic disease. There are no comprehensive guidelines on an ideal strategy for using an ASW, but we have proved the feasibility of our technique in whole-body scans that can be used in clinical practice. We expect that this technique will be clinically applied and will have an impact on lesion detection.

\section{CONCLUSION}

Incorporation of the ASW into the acquisition protocol for bone scintigraphy presents an important tool in scan optimization. We expect that this technique will be clinically used and have an impact on lesion detection. Our study demonstrated that using an ASW was preferred by radiologists interpreting bone scintigraphy, in some cases in a way that was judged to be clinically important. Further research is necessary to determine the influence of using an ASW in obese patients.

\section{DISCLOSURE}

No potential conflict of interest relevant to this article was reported.

\section{ACKNOWLEDGMENT}

We gratefully acknowledge the assistance of our nuclear medicine technologists with data acquisition.

\section{REFERENCES}

1. Hart D, Wall BF. A survey of nuclear medicine in the UK in 2003/04. Gov.UK website. https://www.gov.uk/government/uploads/system/uploads/attachment_data/ file/340207/HpaRpd003.pdf. Published June 2005. Accessed November 20, 2019.

2. Delbeke D, Segall M. Status of and trends in nuclear medicine in the United States. J Nucl Med. 2011;52(suppl):24S-28S.

3. Naddaf SY, Collier D, Elgazzar A, et al. Technical errors in planar bone scanning. J Nucl Med Technol. 2004;32:148-153.

4. Wilson MA. The effect of age on the quality of bone scans using technetium99m pyrophosphate. Radiology. 1981;139:703-705.

5. Nikpoor N. Scintigraphy of the musculoskeletal system. In: Weissman B, eds. Imaging of Arthritis and Metabolic Bone Disease. Philadelphia, PA: Mosby; 2009:17.
6. Potsaid MS, Guiberteau M, McKusick K. Quality of bone scans compared with time between dose and scan. J Nucl Med. 1977;18:787-789.

7. Ulmert D, Solnes L, Thorek D. Contemporary approaches for imaging skeletal metastasis. Bone Res. 2015;3:15024.

8. Merrick M. The normal bone scan. In: Fogelman I, ed. Bone Scanning in Clinical Practise. London, U.K.: Springer; 1987:19-29.

9. Kim DA, Farrell M. It's about time we think about lowering radiation dose in obese patients too. J Nucl Cardiol. 2017;24:1922-1925.

10. Zaidi H, Koral K. Scatter correction strategies in emission tomography. In: Zaidi H. Quantitative Analysis in Nuclear Medicine Imaging. Boston, MA: Springer; 2006:205.

11. Saffar M, Oloomi S, Knoll P, et al. A new approach to scatter correction in SPECT images based on Klein_Nishina equation. Iran J Nucl Med. 2013; 21:19-25.

12. Prekeges J. Image characteristics and performance measures in planar imaging. In: Prekeges J, ed. Nuclear Medicine Instrumentation. Burlington, MA: Jones and Bartlet; 2012:92-93.

13. Nguyen M, Faye C, Eglin L, et al. Apparent image formation by Comptonscattered photons in gamma-ray imaging. IEEE Signal Processing Letters. 2001;8:248-251.

14. Nguyen MK, Truong T, Morvidone M, et al. Scattered radiation emission imaging: principles and applications. Int J Biomed Imaging. 2011;2011:913893.

15. Hijazi H, Magné N, Levy A, et al. Features of cancer management in obese patients. Crit Rev Oncol Hematol. 2013;85:193-205.

16. Wells K, Tumian A, Zapros A, et al. Optimal energy window selection for scintigraphy \& emission computed tomography. CORE website. https://core.ac.uk/download/pdf/19551022.pdf. Accessed November 20, 2019.

17. Stabin M. Data acquisition, reduction, and reporting for nuclear-counting instrumentation [chapter section]. In: Radiation Protection and Dosimetry: An Introduction to Health Physics. Nashville, TN: Vanderbilt University; 2007.

18. Van den Wyngaert T, Strobel K, Kampen W, et al. The EANM practice guidelines for bone scintigraphy. Eur J Nucl Med Mol Imaging. 2016;43:17231738 .

19. The SNM procedure guideline for general imaging 6.0. SNMMI website. http:// snmmi.files.cms-plus.com/docs/General_Imaging_Version_6.0.pdf. Revised 2010. Accessed November 20, 2019.

20. International Atomic Energy Agency. IAEA Quality Control Atlas for Scintillation Camera Systems. Vienna, Austria: IAEA; 2003.

21. Hutton BF, Buvat I, Beekman F. Review and current status of SPECT scatter correction. Phys Med Biol. 2011;56:R85-R112.

22. King MA, Hademenos G, Glick S. A dual-photopeak window method for scatter correction. J Nucl Med. 1992;33:605-612.

23. Collier BD, Palmer D, Knobel J, et al. Gamma camera energy windows for Tc$99 \mathrm{~m}$ bone scintigraphy: effect of asymmetry on contrast resolution. Radiology. 1984;151:495-497.

24. Asgari A, Ashoor M, Sohrabpour M, et al. Evaluation of various energy windows at different radionuclides for scatter and attenuation correction in nuclear medicine. Ann Nucl Med. 2015;29:375-383.

25. Graham LS, LaFontaine RL, Stein MA. Effects of asymmetric photopeak windows on flood field uniformity and spatial resolution of scintillation cameras. J Nucl Med. 1986;27:706-713.

26. Clinical guideline for bone scintigraphy. British Nuclear Medicine Society website. https://cdn.ymaws.com/www.bnms.org.uk/resource/resmgr/guidelines/bnms_bone_ scintigraphy.cf_14.pdf. Published 2014. Accessed February 19, 2020. 